\title{
Histopathological and Immunohistochemical Evaluation of 84 Uterine Sarcomas According to Current Literature
}

\author{
(1) Seda DUMAN ÖZTÜRK,' (1) Gupse TURAN, ${ }^{2}$ (1) Sevgiye KAÇAR ÖZKARA² \\ 'Department of Pathology, Rize Recep Tayyip Erdogan University Training and Research Hospital, Rize-Turkey \\ ${ }^{2}$ Department of Pathology, Kocaeli University Faculty of Medicine Hospital, Kocaeli-Turkey
}

\begin{abstract}
OBJECTIVE
It was aimed to reevaluate uterine sarcoma cases diagnosed in our institution within the last 20 years according to current information and to provide intra-departmental standardization in the differential diagnosis and reporting.

\section{METHODS}

Totally 84 uterine sarcoma cases were re-examined. Recurrence, metastasis, and prognostic data were collected from the patient files. Immunohistochemistry panel consisting of CD10, h-caldesmon, and cyclin D1 was applied to the representative tumor tissues. The relationships between the parameters studied were evaluated statistically.

\section{RESULTS}

Statistically significant differences were found between different histopathological types of uterine sarcomas (US) in terms of age distribution, tumor diameter, mitotic index, necrosis, depth of myometrial invasion, cervical, adnexial and/or omental involvement, lymphovascular invasion, lymph node metastasis, recurrence, and distant metastasis. Statistically significant correlation was determined between the prognosis of the patients and mitotic activity of their tumors and the presence of distant metastasis. The immunohistochemistry panel was found to have significant contribution to the histomorphological examination in the differential diagnosis.
\end{abstract}

\section{CONCLUSION}

Routine use of CD10, h-caldesmon, and cyclin D1 in the histopathological evaluation of US and establishing an updated standard checklist was thought to be beneficial for the differential diagnosis and reporting prognostic parameters.

Keywords: Adenosarcoma; carcinosarcoma; endometrial stromal sarcoma; leiomyosarcoma; undifferentiated uterine sarcoma.

Copyright $\odot$ 2022, Turkish Society for Radiation Oncology

\section{Introduction}

Uterine sarcomas (US) consist of a malignant mesenchymal tumor group, representing $2-6 \%$ of all uterine malignancies.[1,2] The most common histopathological subtype is leiomyosarcomas (LMS) while the second most common is endometrial stromal sarcomas (ESS). LMS has spindle cell, epithelioid and myxoid subtypes. Endometrial stroma and associated tumors are classified as endometrial stromal nodule, low-grade ESS (LGESS), high-grade ESS (HGESS), and undifferentiated uterine sarcoma (UUS). Remaining sub- 
types such as pure heterologous sarcomas, including perivascular epithelioid cell neoplasm (PEComa) and rhabdomyosarcomas, are much less common.[2-4] In addition to these, adenosarcomas (AS) and carcinosarcomas Malignant mixed Mullerian tumors (MMMT) are considered among other US.

Carcinosarcoma has been reclassified as a differentiated or metaplastic form of endometrial carcinoma based largely on its invasion pattern. However, since carcinosarcoma exhibits more aggressive behavior than classical endometrial carcinoma, besides being classified separately as mixed epithelial and mesenchymal tumors according to the 2014 WHO classification, it is still among USs in most retrospective studies.[5,6]

The strongest prognostic factor in USs is reported as stage. In addition, factors such as age, tumor diameter, mitosis and necrosis are also associated with prognosis. Due to the rarity of USs, their evaluation in prospective randomized studies is limited. There are limited case series in the literature that includes all USs.

Our retrospective study provides the advantage of redefining the cases between 1998 and 2014 in terms of subtyping. If this study was planned prospectively, the estimated time to reach statistically sufficient number of cases in terms of diagnosis and prognostic factors would be too long.[1,7] Therefore, we re-evaluated our case series of the last 20 years according to the current diagnostic criteria.

In our institution, immunohistochemical tests such as CD10, cyclin D1, h-caldesmon, p53 were not used routinely in subtyping USs. While evaluating these cases, we completed cases for which immunohistochemical examinations were incomplete. Thus, all the cases evaluated were classified as current and standardized.

While the treatment methods used were only surgery in the past, the guidance of the pathology report on this issue is more important with the inclusion of chemotherapy and radiotherapy among the current treatments. Immunohistochemical examinations may be more important not only for subtyping but also for future treatment of patients. According to the current literature, it was thought that such a study would contribute to the literature in terms of retrospective subtyping in USs, the place of immunohistochemical studies in differential diagnosis and the information that should be included in the pathology report as prognostic parametres.

\section{Materials and Methods}

Eighty-four USs that were stored in our archive for the past 20 years under appropriate conditions were included in the study. All of our cases that meet the study criteria were included in the study; the number of groups and samples that can be evaluated statistically has been reached.

Eighty-four cases were re-examined histomorphologically; tumor diameter, necrosis, mitosis, heterologous elements, myometrial invasion, cervix uteri, ovaries and tubas, omentum were evaluated. leiomyosarcomas (LMS), LGESS, HGESS, UUS, AS, and MMMT cases were grouped as younger or older than 50 years of age.

While the tumor diameter was determined as $<5 \mathrm{~cm}$, between 5 and $10 \mathrm{~cm}$ and $>10 \mathrm{~cm}$; values of $<10,10-20$ and $>20 / 10$ high power fields (HPF) were used for mitotic figures. When choosing these values, current literature information was taken into consideration.

A representative paraffin block of the tumor was selected from all cases for immunohistochemical examinations. Immunohistochemical studies were performed in the automated Ventana Benchmark XT closed device using h-caldesmon (caldesmon [monoclonal], CellMarque, 1: 100 dilution); anti-CD10 (anti CD10 [monoclonal], Ventana, 1: 100 dilution) and cyclin D1 (anti-Cyclin D1 [monoclonal], Ventana, 1: 100 dilution) antibodies.

Histopathological and immunohistochemical evaluation of the cases was made without knowing the diagnosis in order to prevent possible "bias."

\section{Results}

In our study, out of 84 cases, which were histopathologically re-evaluated according to the current literature, 19 were LGESS, nine were HGESS, 23 were LMS, 10 were UUS, 20 were MMMT, and three were AS.

The average age of the patients included in the study was 55.9 (SD: 13.26). Patients were minimum 20 -years-old and maximum 81-years-old. Of the cases; 29 of them were under the age of 50, 55 of them were in the age group of 50 and over. The highest mean age (65.4 years) was found in the group with MMMT diagnosis $(\mathrm{p}=0.000)$ (Table 1$)$.

Tumor diameters varied between 1.3 and $18 \mathrm{~cm}$ for ESS, 4.5 and $68 \mathrm{~cm}$ for LMS, 4 and $22.5 \mathrm{~cm}$ for UUS, 1.7 and $15 \mathrm{~cm}$ for MMMT, and 4 and $22 \mathrm{~cm}$ for AS. It was observed that $90 \%$ of the cases in the UUS group had a tumor diameter over $10 \mathrm{~cm}$. The tumor diameter was $<5 \mathrm{~cm}$ in $63.2 \%$ of the cases in the LGESS group $(\mathrm{p}=0.001)$ (Table 1).

There was a statistically significant difference in mitotic count between LGESS and other high-grade 
Table 1 Statistically significant clinicopathological parameters between the histopathological types of uterine sarcomas

\begin{tabular}{|c|c|c|c|c|c|c|c|}
\hline $\begin{array}{l}\text { Clinico-pathological } \\
\text { parameters }\end{array}$ & $\begin{array}{l}\text { LGESS } \\
\text { (\%) }\end{array}$ & $\begin{array}{c}\text { HGESS } \\
\text { (\%) }\end{array}$ & $\begin{array}{l}\text { LMS } \\
(\%)\end{array}$ & $\begin{array}{l}\text { UUS } \\
\text { (\%) }\end{array}$ & $\begin{array}{l}\text { MMMT } \\
(\%)\end{array}$ & $\begin{array}{l}\text { AS } \\
(\%)\end{array}$ & $\mathbf{p}$ \\
\hline \multicolumn{8}{|l|}{ Age } \\
\hline$<50$ & 68.4 & 11.1 & 34.8 & 30 & 10 & 66.7 & 0.001 \\
\hline$\geq 50$ & 31.6 & 88.9 & 65.2 & 70 & 90 & 33.3 & \\
\hline \multicolumn{8}{|l|}{ Tumor size } \\
\hline$\leq 5 \mathrm{~cm}$ & 63.2 & 33.3 & 13 & 0 & 25 & 33.3 & 0.001 \\
\hline $5-10 \mathrm{~cm}$ & 15.8 & 55.6 & 34.8 & 10 & 45 & 33.3 & \\
\hline$>10 \mathrm{~cm}$ & 21.1 & 11.1 & 52.2 & 90 & 30 & 33.3 & \\
\hline \multicolumn{8}{|l|}{ Mitotic index } \\
\hline$\leq 10$ & 84.2 & 0 & 26.1 & 10 & 15 & 33.3 & 0.008 \\
\hline $10-20$ & 5.3 & 33.3 & 17.4 & 30 & 25 & 0 & \\
\hline$>20$ & 10.5 & 66.7 & 56.5 & 60 & 60 & 66.7 & \\
\hline \multicolumn{8}{|l|}{ Necrosis } \\
\hline Absent & 68.4 & 33.3 & 13 & 0 & 60 & 66.7 & 0.000 \\
\hline Present & 31.6 & 66.7 & 87 & 100 & 40 & 33.3 & \\
\hline \multicolumn{8}{|l|}{ Myometrial invasion } \\
\hline$\leq 1 / 2$ & 62.5 & 12.5 & 22.2 & 10 & 35 & 0 & 0.024 \\
\hline$>1 / 2$ & 37.5 & 87.5 & 77.8 & 90 & 65 & 100 & \\
\hline \multicolumn{8}{|l|}{ Cervical invasion } \\
\hline Absent & 100 & 60 & 86.4 & 60 & 55 & 66.7 & 0.025 \\
\hline Present & 0 & 40 & 13.6 & 40 & 45 & 33.3 & \\
\hline \multicolumn{8}{|c|}{ H-Kaldesmon immuno-reactivity } \\
\hline Negative & 100 & 100 & 52.2 & 90 & 95 & 100 & 0.008 \\
\hline Focally positive & 0 & 0 & 13 & 0 & 0 & 0 & \\
\hline Diffusely positive & 0 & 0 & 34.8 & 10 & 5 & 0 & \\
\hline \multicolumn{8}{|c|}{ CD 10 immuno-reactivity } \\
\hline Negative & 26.3 & 55.6 & 95.7 & 40 & 80 & 66.7 & 0.002 \\
\hline Focally positive & 21.1 & 22.2 & 0 & 20 & 5 & 0 & \\
\hline Diffusely positive & 52.6 & 22.2 & 4.3 & 40 & 15 & 33.3 & \\
\hline \multicolumn{8}{|c|}{ Cyclin D1 immuno-reactivity } \\
\hline Negative & 89.5 & 22.2 & 91.3 & 90 & 80 & 66.7 & 0.002 \\
\hline Focally positive & 0 & 22.2 & 0 & 0 & 15 & 33.3 & \\
\hline Diffusely positive & 10.5 & 55.6 & 8.7 & 10 & 5 & 0 & \\
\hline
\end{tabular}

LGESS: Low grade endometrial stromal sarcomas; HGESS: High grade endometrial stromal sarcomas; LMS: Leiomyosarcomas; UUS: Undifferentiated uterine sarcomas; MMMT: Malignant mixed Mullerian tumors; AS: Adenosarcomas

sarcomas. High-grade sarcomas had higher mitotic counts. Mitotic index was $>20 / 10 \mathrm{HPF}$ in $48.8 \%$ of our cases. In the LGESS group, it was noted that the mitotic index was $\leq 15 / 10 \mathrm{HPF}$ in $89.5 \%$ of the cases $(\mathrm{p}=0.008)$ (Table 1$)$.

Tumor necrosis was observed in $66.7 \%(51 / 84)$ of our cases. It was seen in all cases with UUS (100\%) diagnosis, in 20 cases with LMS (87\%), in eight cases with the diagnosis of MMMT (40\%), and in one of the cases with the diagnosis of adenosarcoma (33.3\%). The sarcoma subgroup in which tumor necrosis was most common was UUS followed by LMS ( $\mathrm{p}=0.000$ ) (Table 1 ).

Histopathological evaluation in terms of myometrial invasion could be done in 73 cases. While deep myo- metrial invasion was detected in $68.5 \%$ (50/73) of total cases; superficial myometrial invasion was observed in $62.5 \%(10 / 16)$ of the cases in the LGESS group. Deep myometrial invasion was found in $6(37.5 \%)$ cases with LGESS; in 7 (87.5\%) of the HGESS cases; in $14(77.8 \%)$ of the LMS cases; in 9 (90\%) of the UUS cases; in 13 (65\%) of the MMMT cases and in 1 of the cases diagnosed with adenosarcoma ( $\mathrm{p}=0.024)$ (Table 1$)$.

Cervix uteri involvement was observed in two of the cases diagnosed with HGESS; in three of the cases diagnosed with LMS; in four of the cases with UUS diagnosis; in 9 of the cases with MMMT diagnosis; in one of the cases diagnosed with adenosarcoma. Cervical stromal invasion was detected in $45 \%$ (9/20) of our 
MMMT patients and in $40 \%$ of our patients diagnosed with HGESS (2/5) and UUS (4/10). Cervical involvement was not observed in any of our patients with a diagnosis of LGESS ( $\mathrm{p}=0.025$ ) (Table 1 ).

In terms of the stages of the cases versus histopathological diagnoses; our cases were in stage IV disease with a rate of $17.9 \%(15 / 84)$. It was observed that these patients were predominantly in the UUS, MMMT and LMS groups $(\mathrm{p}=0.123)$ (Table 1$)$.

In our study, the follow-up periods of our patients ranged from 2 to 152 months; the average follow-up time was 27.5 months (SD: 32.7 ). Ten patients died during this follow-up period. While a mitotic figure of $>10 / 10$ HPF was observed in histopathological examination in 10 of our patients who died, 9 of them had deep myometrial invasion.

A statistically significant difference was found between the mean survival time (114.9 months) of the patients in the stage I-II group and the mean survival time (91.5 months) of the patients in the stage III-IV group ( $\mathrm{p}=0.026)$ (Table 1$)$.

Coming to the relationship between distant organ metastases and survival of the cases; a statistically significant correlation was found between the prognosis of the patients and the presence/absence of distant organ metastases $(\mathrm{p}=0.005)$ (Table 2). Distant metastasis was present in $6(60 \%)$ of 10 patients who died during the follow-up period. In the group of 57 cases without distant metastasis, only 4 patients (7\%) died.

Lymphovascular invasion was observed in 17 (20.2\%) cases. The diagnostic groups in which LVI was most frequently observed were MMMT (35\%) and LGESS (26.3\%) ( $\mathrm{p}=0.33)$ (Table 1).

Last but not the least immunohistochemical findings were evaluated strong and diffuse immunoreactivity was detected with h-caldesmon in $34.8 \%(8 / 23)$ of the LMS cases, and focal positive immunoreactivity in $3(13 \%)$ of them. $\mathrm{H}$-caldesmon expression was observed in one case of each from the UUS and MMMT

\begin{tabular}{lcccc}
\hline Table 2 & \multicolumn{6}{l}{$\begin{array}{l}\text { Relationship between survival of the cases ver- } \\
\text { sus mitotic index and distant organ metastases }\end{array}$} \\
$\begin{array}{l}\text { Clinico- } \\
\text { pathological }\end{array}$ & Parameters & $\begin{array}{c}\text { Alive } \\
\text { (\%) }\end{array}$ & $\begin{array}{c}\text { Exitus } \\
\text { (\%) }\end{array}$ & p \\
\hline Mitotic index & $\leq 10 / 10$ HPF & 34.4 & 0 & $\mathbf{0 . 0 2 8}$ \\
\multirow{2}{*}{$\begin{array}{l}\text { Distant metastasis } \\
\end{array}$} & Absent & 84.4 & 40 & $\mathbf{0 . 0 0 5}$ \\
& Present & 15.6 & 60 & \\
\hline
\end{tabular}

HPF: High power fields groups. No positivity was observed in any case of the ESS group ( $\mathrm{p}=0.008)$ (Table 1).

With CD10 staining, 52.6\% (10/19) of LGESSs showed diffuse and strong positivity while $21.1 \%$ (4/19) showed focal positive immunoreactivity. CD10 expression was remarkable in 4 patients in the UUS group, 3 patients in the MMMT group, and 1 patient of the each LMS and AS groups among other diagnosis groups $(\mathrm{p}=0.002)$ (Table 1$)$.

Cyclin D1 positivity was observed in $50 \%$ of the cases detected at advanced stage. With cyclin D1, 55.6\% (5/9) of HGESS cases showed diffuse and strong ( $>70 \%$ cells) positive immunoreaction. There were rare positive cases in other diagnostic groups $(\mathrm{p}=0.002)$ (Table 1).

Diffuse/strong positive intranuclear immunoreactivity with p53 was detected in $50 \%(11 / 22)$ of the cases in the LMS group. However, no significant correlation was observed between p53 immunoreactivity and survival of the patients in this group.

The histopathological/immunohistochemical results of our cases that were found to be statistically significant are summarized in Table 1.

Examples of different histopathological subtypes of USs and immunohistochemical results are presented in Figures 1-6.

\section{Discussion}

USs consist of an uncommon malignant mesenchymal tumor group representing 2-6\% of all uterine malignancies.[1,2] The most common histopathological subtype is LMS, which constitutes $60 \%$ of US cases, and ESS the second most common with $20-25 \%$ of incidence.[2] In the present study, there were LMS cases in the first order and LGESS and HGESS cases were in the second order.

Most of the series in the literature consist of limited number of patients. This is because USs are rare. In two large reviews by D'Angelo and Prat (2010) and Gadducci et al. (2011),[5,8] it is reported that USs constitute $3-7 \%$ of uterine cancers. For this reason, in the current literature, there is no consensus yet on the prognostic factors of USs, hence clinical results and treatment strategies.

The largest series published to date on USs is the study of Nordal et al.[9] In this study, the incidence and survival of USs were investigated by following the Norwegian female population for 37 years. In another study of Norwegian origin, by Abeler et al.[10] publishing detailed histopathological and survival data of 419 


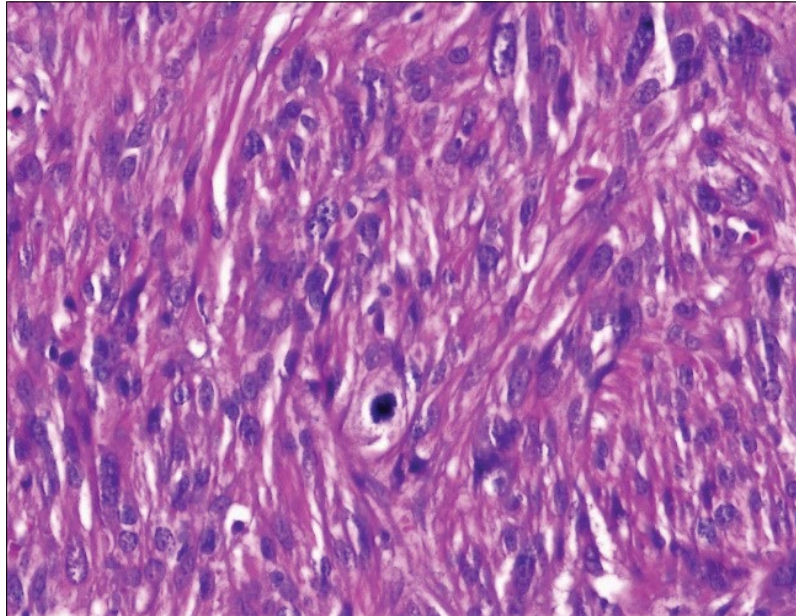

Fig. 1. Leiomyosarcoma, $(\mathrm{H} \& \mathrm{E} \times 400)$.

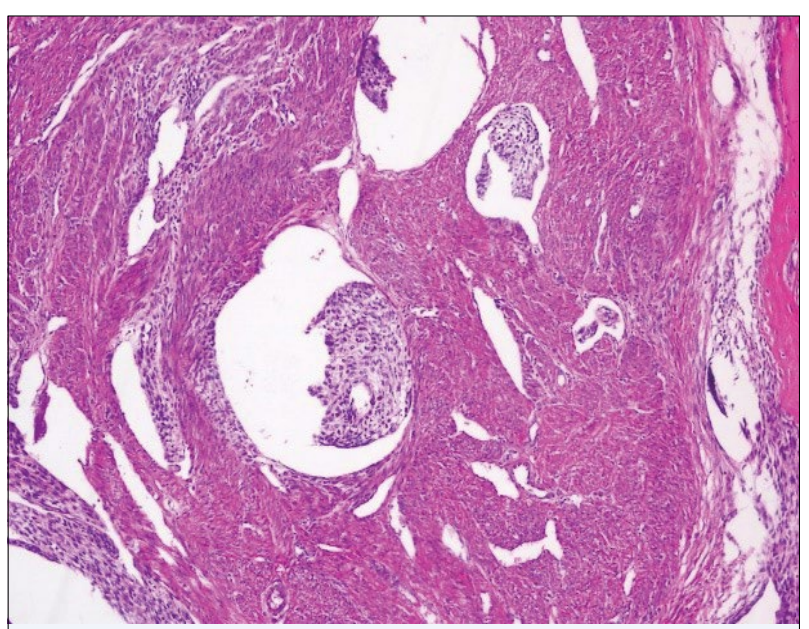

Fig. 2. Lymphovascular invasion in LGESS, (H\&E×100). LGESS: Low grade endometrial stromal sarcomas.

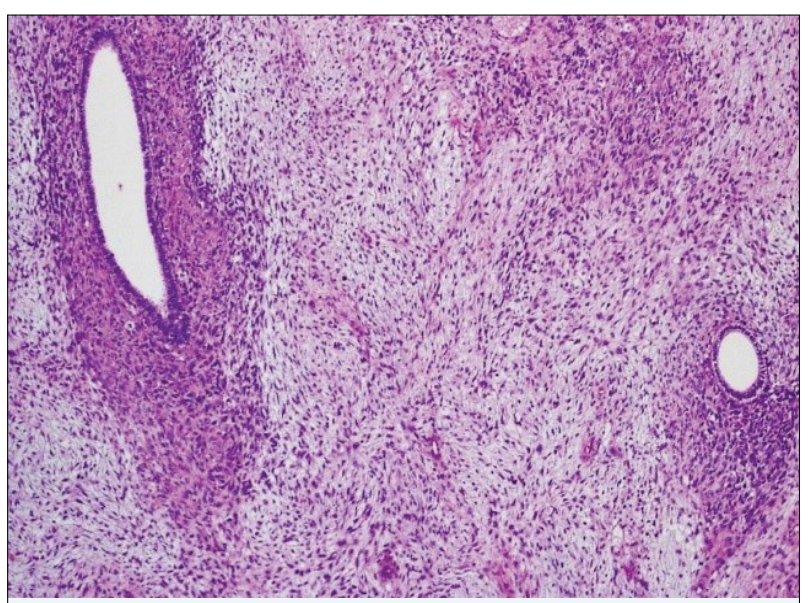

Fig. 3. Adenosarcoma, $(\mathrm{H} \& \mathrm{E} \times 200)$.

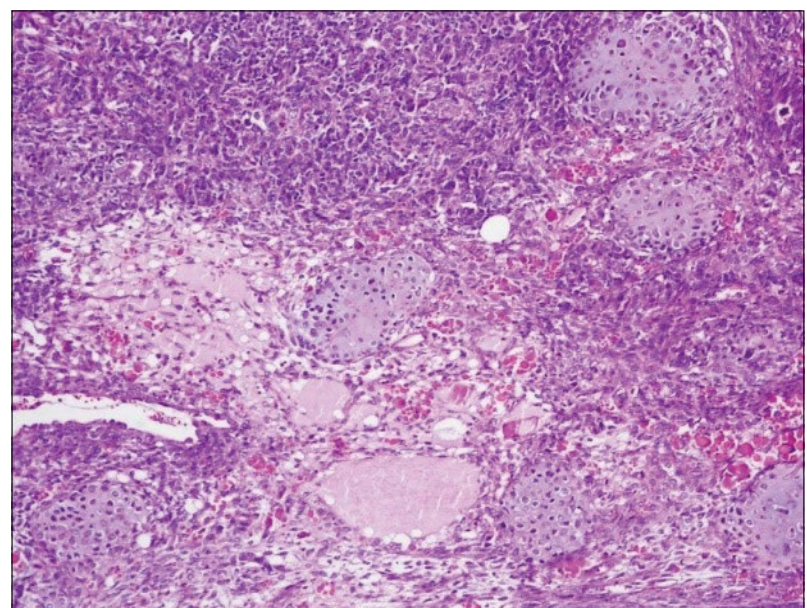

Fig. 4. Carcinosarcoma, $(\mathrm{H} \& \mathrm{E} \times 100)$.

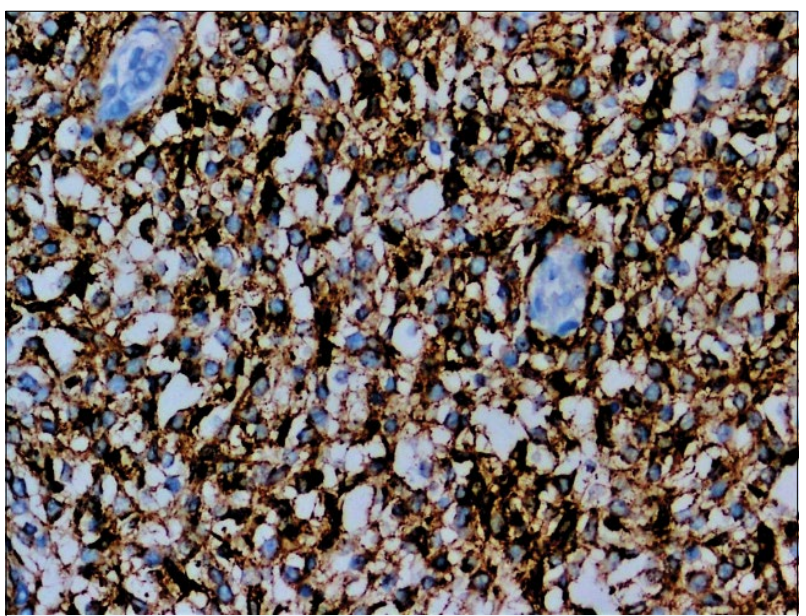

Fig. 5. CD10 in LGESS (IHC $\times 400)$.

LGESS: Low grade endometrial stromal sarcomas.

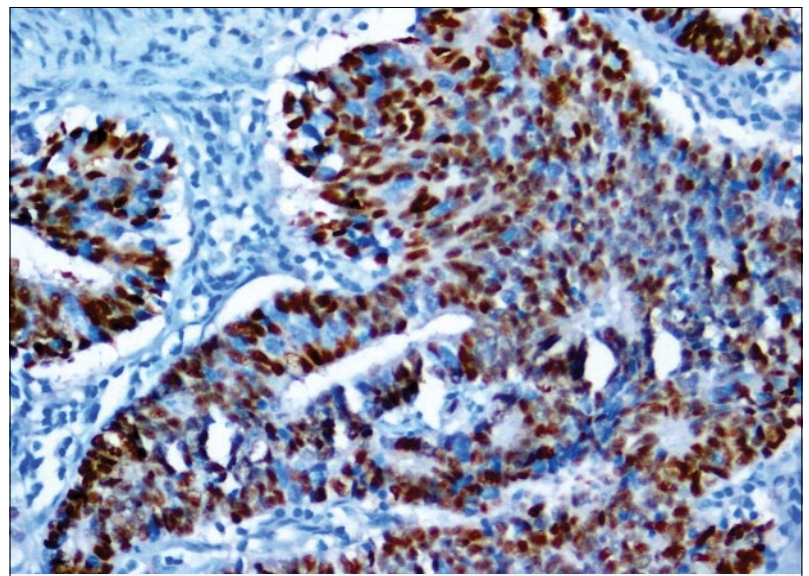

Fig. 6. Cyclin D1 in HGESS (IHC $\times 400)$.

HGESS: High grade endometrial stromal sarcomas. 
cases with known prognosis and documenting the risk factors of each diagnostic group, they reported that stage was the strongest prognostic factor. In the present study, a statistically significant difference was found between the mean survival time 114.9 months of the patients in the stages I-II group and the mean survival time (91.5 months) of the patients in the stages III-IV group.

Considering the age distribution of USs, it is noteworthy that they generally occur in advanced ages. In the study conducted by Gao et al., the mean age in the premenopausal group of ESS was 45; MMMT has always been reported in the age of 50 or over.[7,11] In accordance with the literature, the mean age was 47.8 in our LGESS cases, 56 in the HGESS group, and 65.4 in the MMMTs out of 84 US cases we examined.

There are studies in the literature showing that tumor diameter is related to prognosis in USs.[10,12] It has been reported that the size of the tumor has a prognostic value, especially in stage I disease.[13-15] In our study, 33 out of all USs had a tumor diameter $>10 \mathrm{~cm}$. Tumor diameter was larger than $10 \mathrm{~cm}$ in 6 out of 10 patients who died. While cases with high tumor diameter may be at low stage, none of the cases with low tumor diameter were at advanced stage. As a matter of fact, FIGO staging took the tumor diameter $<5 \mathrm{~cm}$ in USs as stage I criteria.

Both parameters are valuable for differential diagnosis when USs are evaluated in terms of the number of mitoses and necrosis they contain. High mitotic activity ( $>10 / 10 \mathrm{HPF})$ is more common in UUS and LMS. High mitotic activity is a factor used for differential diagnosis in USs and associated with poor prognosis. Again, the absence of necrosis in LGESS is a valuable parameter used in the differential diagnosis of USs. In our study, all of the cases died during the follow-up period had high mitotic activity.

The depth of myometrial invasion has also an important effect on prognosis. $[7,16]$ The depth of myometrial invasion is also included in FIGO staging of AS and carcinosarcomas. In terms of myometrial invasion in our series, deep myometrial invasion was detected in $68.5 \%(50 / 73)$ in cases with histopathological evaluation; superficial myometrial invasion was observed in $62.5 \%(10 / 16)$ of the cases in the LGESS group while deep myometrial invasion was present in $90 \%$ of the patients who died.

Most studies on USs highlight the stage as the most important prognostic factor.[7-9,17-21] Five-year survival rates have been reported to range from $29 \%$ to $74 \%$ for stage I and between 20 and $40 \%$ for other stages.[18,22,23] In the series presented by Kokawa et al. in terms of prognosis by stage, there was a similar rate (52\%) in stage III and IV, as in the data (50\%) in the study by Gao et al. [7,24] In the study conducted by Gao et al. 63.6\% of the patients in the LMS group were diagnosed at stage I-II; and those in the MMMT group at more advanced stages (stage III-IV, 66.6\%). In our study, a statistically significant difference was found between the mean survival time (114.9 months) of the patients in the stage I-II group and that (91.5 months) of the patients in the stage III-IV group.

In the study of Pautier et al.[25] regardless of the stage and histopathological subtype, the diagnosis of US itself confirms the poor prognosis. It was stated that only LGESS has a favorable prognosis with surgery alone and does not require further therapeutic interventions. It was also emphasized that age, in addition to stage, is a strong prognostic factor for MMMT.

Comparison of IHC analysis in different studies on USs is still open to debate. The contradictions in immunoreactivity results reported in many studies might be the result of the variability of antibody clones, dilutions of antibodies, different staining techniques, and different patient populations. In addition, threshold values for positive immunoreactivity are not always clearly defined in the literature and therefore results may be variable.[26]

Lee et al.[27] in their works published in 2012, suggested that the presence of myopermeatic growth pattern in a uterine tumor consisting of cyclin D1 positive, non-CD10/ER/PR expressing, high grade, monomorphic round cells indicates ESS with YWHAE-FAM22 genetic fusion. In this study, diffuse ( $>70 \%)$ and strong cyclin D1 immunoreactivity was also observed in the UUS with uniform nucleus (UUS-U) subgroup and rarely in cases of uterine leiomyosarcoma. However, YWHAE (or JAZF1/PHF1) regulations were not detected in these by FISH analysis. Importantly, cyclin D1 positive UUS-U cases also showed diffuse strong CD10 immunoreactivity, unlike round cell component of HGESS with the genetic fusion of YWHAE-FAM22, which is CD10 negative. Therefore, when cyclin D1 and CD10 immunohistochemistry staining is applied to a histopathologically high-grade but non-pleomorphic uterine sarcoma, it is argued that diffuse strong cyclin D1 staining in the absence of CD10 staining is highly sensitive and specific for HGESS harboring the YWHAE FAM22 genetic fusion.[27]

Emphasizing that the diffuse strong cyclin D1 immunoreactivity was observed in a correlated way in HGESS with YWHAE-FAM22 genetic fusion; a diagnostic algorithm including morphological and immu- 
nophenotypic features is proposed to identify ESS with uterine YWHAE-FAM22 genetic fusion.[27] Following this study, in the WHO 2014 classification, ESSs were again divided into LGESSs and HGESSs.[28] On the other hand, the mechanism of cyclin D1 overexpression in ESS with YWHAE-FAM22 genetic fusion has not been explained.[27]

Cyclin D1 immunoreactivity has been previously evaluated by Kurihara et al.[29] in both endometrial stromal tumors and UUS. Cyclin D1 immunoreactivity has also been studied in uterine MMMT before.[30,31] De Jong et al.[30] found that 7 out of 31 MMMT cases had cyclin D1 positivity in the mesenchymal component; however, these authors used a much lower (10\%) nuclear staining score threshold.

An important subgroup of Ewing sarcomas can also show diffuse strong nuclear cyclin D1 immunoreactivity.[32] ESS with YWHAE-FAM22 genetic fusion may also show diffuse strong CD99 immunoreaction.[33] This overlap in the immunophenotype creates difficulties in the differential diagnosis when a malignant round cell tumor is encountered in the biopsy of a pelvic mass. Molecular analysis (FISH or Real-time polymerase chain reaction) is required for the differential diagnosis of Ewing sarcoma from ESS with the genetic fusion of YWHAE-FAM22.[27]

In our study, one out of eight cases diagnosed with HGESS and whose prognostic information could be reached was lost during the follow-up period and this case showed negative immunoreaction with cyclin D1. Unfortunately, we have no fascility for molecular genetic studies of our cases at our institution yet.

On the other hand, Koivisto-Korander et al.[26] showed that there was a statistically significant relationship between the p53, ERa and PRa markers and the survival of LMS patients. While $\mathrm{p} 53$ positivity was detected in $30 \%$ of MMMT, $26 \%$ of LMS and $12 \%$ of ESS cases; they reported longer survival in p53-negative LMS cases (median survival time of 123 months compared to 11 months in $\mathrm{p} 53$ positive patients).

In our study, diffuse/strong positive intranuclear p53 immunoreactivity was observed in 50\% (11/22) of the cases in the LMS group. However, there was no statistically significant relationship between p53 immunoreactivity and survival of the patients in this group. The differences between these results might be due to the fact that the studies were performed with a small number of LMS cases and the dilution of antibodies used in immunohistochemical studies.

Chemotherapeutic agents are also used in addition to surgical treatment in Uss.[34] Performing genetic analysis, especially in ESSs, might predict the use of different agents in treatment. The first bicentric study presented on this subject was by Hemming et al. Targeted immunotherapy has also been recently mentioned in the literature. [35,36]

The limitations of this study include the small number of cases, as in other studies, and the necessity to adhere to the incidence in histopathological diagnostic groups. Therefore, it might not be possible for significant prognostic factors found in larger case series to be evident in our case series.

In the light of the results we obtained in our study, an algorithm for the differential diagnosis and a report format for classification and reporting of these cases, which are quite rare in our daily practice, was prepared for the purpose of standardization in our department. In the commentary part of the report, it was recommended to re-emphasize the risk factors associated with case-specific recurrence and prognosis. In addition, a genetic study was planned for molecular investigation.

Peer-review: Externally peer-reviewed.

Conflict of Interest: All authors declared no conflict of interest.

Ethics Committee Approval: The study was approved by The Kocaeli University Non-Invasive Clinical Research Ethics Committee (No: KÜ GOKAEK 2018/20.18, Date: 26/12/2018).

Financial Support: This study has received no financial support.

Authorship contributions: Concept - S.K.Ö.; Design S.D.Ö., S.K.Ö.; Supervision - S.D.Ö., S.K.Ö., G.T.; Funding - S.D.Ö., S.K.Ö.; Materials - S.D.Ö., S.K.Ö., G.T.; Data collection and/or processing - S.D.Ö., S.K.Ö., G.T.; Data analysis and/or interpretation - S.D.Ö., S.K.Ö.; Literature search - S.D.Ö., S.K.Ö.; Writing - S.D.Ö., S.K.Ö.; Critical review S.D.Ö., S.K.Ö.

\section{References}

1. Santos P, Cunha TM. Uterine sarcomas: Clinical presentation and MRI features. Diagn Interv Radiol 2015;21(1):4-9.

2. Barquet-Muñoz SA, Isla-Ortiz D, Montalvo-Esquivel G, Cantú-de-León D, Salcedo-Hernández RA, Cordoba-Gonzalez V, et al. Prognostic factors associated with uterine sarcomas: The experience of a single institution. J Obstet Gynaecol 2019;39(2):231-6.

3. El-Khalfaoui K, du Bois A, Heitz F, Kurzeder C, Se- 
houli J, Harter P. Current and future options in the management and treatment of uterine sarcoma. Ther Adv Med Oncol 2014;6(1):21-8.

4. Fadare O. Heterologous and rare homologous sarcomas of the uterine corpus: A clinicopathologic review. Adv Anat Pathol 2011;18(1):60-74.

5. D’Angelo E, Prat J. Uterine sarcomas: A review. Gynecol Oncol 2010;116(1):131-9.

6. Mallmann P. Uterine sarcoma-difficult to diagnose, hard to treat. Oncol Res Treat 2018;41(11):674.

7. Gao Y, Meng H, Zhang Y, Jiao T, Hui N. Retrospective analysis of 80 cases with uterine carcinosarcoma, leiomyosarcoma and endometrial stromal sarcoma in China, 1988-2007. Int J Clin Exp Pathol 2014;7(4):1616-24.

8. Gadducci A, Landoni F, Sartori E, Zola P, Maggino T, Lissoni A, et al. Uterine leiomyosarcoma: Analysis of treatment failures and survival. Gynecol Oncol 1996;62(1):25-32.

9. Nordal RR, Kristensen GB, Stenwig AE, Nesland JM, Pettersen EO, Trope CG. An evaluation of prognostic factors in uterine carcinosarcoma. Gynecol Oncol 1997;67(3):316-21.

10. Abeler VM, Røyne O, Thoresen S, Danielsen HE, Nesland JM, Kristensen GB. Uterine sarcomas in Norway. A histopathological and prognostic survey of a total population from 1970 to 2000 including 419 patients. Histopathology 2009;54(3):355-64.

11. Günthert AR. Sarcomas and mixed mesodermal tumors of the uterus. Therapeutische Umschau. Ther Umsch 2011;68(10):559-64.

12. Leitao MM Jr., Zivanovic O, Chi DS, Hensley ML, O'Cearbhaill R, Soslow RA, et al. Surgical cytoreduction in patients with metastatic uterine leiomyosarcoma at the time of initial diagnosis. Gynecol Oncol 2012;125(2):409-13.

13. Larson B, Silfverswärd C, Nilsson B, Pettersson F. Prognostic factors in uterine leiomyosarcoma. A clinical and Histopathological study of 143 cases. The Radiumhemmet series 1936-1981. Acta Oncol 1990;29(2):185-91.

14. Evans HL, Chawla SP, Simpson C, Finn KP. Smooth muscle neoplasms of the uterus other than ordinary leiomyoma. A study of 46 cases, with emphasis on diagnostic criteria and prognostic factors. Cancer 1988;62(10):2239-47.

15. Nordal RR, Kristensen GB, Kaern J, Stenwig AE, Pettersen EO, Tropé CG. The prognostic significance of stage, tumor size, cellular atypia and DNA ploidy in uterine leiomyosarcoma. Acta Oncol 1995;34(6):797802.

16. Park JY, Park SK, Kim DY, Kim JH, Kim YM, Kim YT, et al. The impact of tumor morcellation during surgery on the prognosis of patients with apparently early uterine leiomyosarcoma. Gynecol Oncol 2011;122(2):255-9.

17. Wolfson AH, Wolfson DJ, Sittler SY, Breton L, Markoe AM, Schwade JG, et al. A multivariate analysis of clinicopathologic factors for predicting outcome in uterine sarcomas. Gynecol Oncol 1994;52(1):56-62.

18. Salazar OM, Bonfiglio TA, Patten SF, Keller BE, Feldstein M, Dunne ME, et al. Uterine sarcomas: Natural history, treatment and prognosis. Cancer 1978;42(3):1152-60.

19. Wheelock JB, Krebs HB, Schneider V, Goplerud DR. Uterine sarcoma: Analysis of prognostic variables in 71 cases. Am J Obstet Gynecol 1985;151(8):1016-22.

20. Kahanpää KV, Wahlström T, Gröhn P, Heinonen E, Nieminen U, Widholm O. Sarcomas of the uterus: A clinicopathologic study of 119 patients. Obstet Gynecol 1986;67(3):417-24.

21.Durnali A, Tokluoğlu S, Özdemir N, Inanç M, Alkiş $\mathrm{N}$, Zengin N, et al. Prognostic factors and treatment outcomes in 93 patients with uterine sarcoma from 4 centers in Turkey. Asian Pac J Cancer Prev 2012;13(5):1935-41.

22. Covens AL, Nisker JA, Chapman WB, Allen HH. Uterine sarcoma: An analysis of 74 cases. Am J Obstet Gynecol 1987;156(2):370-4.

23. Kohorn EI, Schwartz PE, Chambers JT, Peschel RE, Kapp DS, Merino M. Adjuvant therapy in mixed mullerian tumors of the uterus. Gynecol Oncol 1986;23(2):212-21.

24. Kokawa K, Nishiyama K, Ikeuchi M, Ihara Y, Akamatsu N, Enomoto T, et al. Clinical outcomes of uterine sarcomas: Results from 14 years worth of experience in the Kinki district in Japan (1990-2003). Int J Gynecol Cancer 2006;16(3):1358-63.

25. Pautier P, Genestie C, Rey A, Morice P, Roche B, Lhommé $\mathrm{C}$, et al. Analysis of clinicopathologic prognostic factors for 157 uterine sarcomas and evaluation of a grading score validated for soft tissue sarcoma. Cancer 2000;88(6):1425-31.

26. Koivisto-Korander R, Butzow R, Koivisto AM, Leminen A. Immunohistochemical studies on uterine carcinosarcoma, leiomyosarcoma, and endometrial stromal sarcoma: Expression and prognostic importance of ten different markers. Tumour Biol 2011;32(3):451-9.

27. Lee CH, Mariño-Enriquez A, Ou W, Zhu M, Ali RH, Chiang S, et al. The clinicopathologic features of YWHAE-FAM22 endometrial stromal sarcomas: A histologically high-grade and clinically aggressive tumor. Am J Surg Pathol 2012;36(5):641-53.

28. Oliva E, Carcangiu ML, Carinelli SG, Young RH. Tumours of the uterine corpus, mesenchymal tumours. In: Kurman RJ, Carcangiu ML, Herrington CS, Young RH, editors. WHO Classification of Tumours of Female Reproductive Organs. $4^{\text {th }}$ ed. Lyon: IARC; 2014. p. 135-45. 29. Kurihara S, Oda Y, Ohishi Y, Kaneki E, Kobayashi H, 
Wake $\mathrm{N}$, et al. Coincident expression of $\beta$-catenin and cyclin D1 in endometrial stromal tumors and related high-grade sarcomas. Mod Pathol 2010;23(2):225-34.

30. de Jong RA, Nijman HW, Wijbrandi TF, Reyners AK, Boezen HM, Hollema H. Molecular markers and clinical behavior of uterine carcinosarcomas: Focus on the epithelial tumor component. Mod Pathol 2011;24(10):1368-79.

31. Kanthan R, Senger JL, Diudea D. Malignant mixed Mullerian tumors of the uterus: Histopathological evaluation of cell cycle and apoptotic regulatory proteins. World J Surg Oncol 2010;8:60.

32. Fuchs B, Inwards CY, Janknecht R. Vascular endothelial growth factor expression is up-regulated by EWSETS oncoproteins and Sp1 and may represent an independent predictor of survival in Ewing's sarcoma. Clin Cancer Res 2004;10(4):1344-53.

33. Amant F, van Calsteren K, Halaska MJ, Beijnen J, La- gae L, Hanssens M, et al. Gynecologic cancers in pregnancy: Guidelines of an international consensus meeting. Rare and Uncommon Gynecological Cancers. Berlin: Springer; 2011. p. 209-27.

34. Benson C, Ray-Coquard I, Sleijfer S, Litière S, Blay JY, Le Cesne A, et al. Outcome of uterine sarcoma patients treated with pazopanib: A retrospective analysis based on two European organisation for research and treatment of cancer (EORTC) soft tissue and bone sarcoma group (STBSG) clinical trials 62043 and 62072. Gynecol Oncol 2016;142(1):89-94.

35. Hemming ML, Wagner AJ, Nucci MR, Chiang S, Wang L, Hensley ML, et al. YWHAE-rearranged highgrade endometrial stromal sarcoma: Two-center case series and response to chemotherapy. Gynecol Oncol 2017;145(3):531-5.

36. Tuyaerts S, Amant F. Endometrial stromal sarcomas: A revision of their potential as targets for immunotherapy. Vaccines (Basel) 2018;6(3):56. 